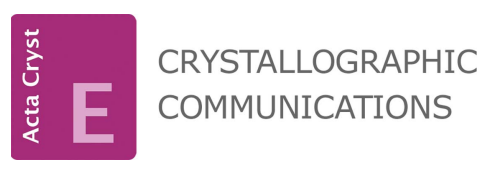

ISSN 2056-9890

Received 20 October 2016

Accepted 24 October 2016

Edited by M. Zeller, Purdue University, USA

Keywords: crystal structure; $\pi-\pi$ stacking;

hydrogen bond; halogen bond.

CCDC reference: 1511202

Supporting information: this article has supporting information at journals.iucr.org/e

\section{Crystal structure of 7-iodo-4-oxo-4H-chromene-3- carbaldehyde}

\author{
Yoshinobu Ishikawa* \\ School of Pharmaceutical Sciences, University of Shizuoka, 52-1 Yada, Suruga-ku Shizuoka 422-8526, Japan. \\ *Correspondence e-mail: ishi206@u-shizuoka-ken.ac.jp
}

In the title compound, $\mathrm{C}_{10} \mathrm{H}_{5} \mathrm{IO}_{3}$, an iodinated 3-formylchromone derivative, the non-H atoms are essentially coplanar (r.m.s. deviation $=0.0344 \AA$ ), with the largest deviation from the least-squares plane [0.101 (3) $\AA$ ] being found for the formyl $\mathrm{O}$ atom. In the crystal, molecules are linked through stacking interactions [centroid-centroid distance between the benzene rings $=3.700$ (3) $\AA$ ] and C$\mathrm{H}$. . O hydrogen bonds. Halogen bonds between the I atoms at 7-position and the formyl $\mathrm{O}$ atoms $\left[\mathrm{I} 1 \cdots \mathrm{O} 3=3.056(2) \AA\right.$, C6-I1 $\cdots \mathrm{O} 3=173.18(8)^{\circ}$ and $\mathrm{I} 1 \cdots \mathrm{O} 3-\mathrm{C} 10=111.12(18)^{\circ}$ ] are also formed along [110], resulting in sheets perpendicular to the $c$ axis, constructed by $\mathrm{C}-\mathrm{H} \cdots \mathrm{O}$ hydrogen bonds and $\mathrm{I} \cdots \mathrm{O}$ halogen bonds.

\section{Chemical context}

3-Formylchromone and its derivatives show versatile biological activities such as anti-inflammatory activity (Khan et al., 2010) and the inhibition of protein tyrosine phosphatase 1B (Shim et al., 2005), thymidine phosphorylase (Khan et al., 2009), carbonic anhydrase (Ekinci et al., 2012), and metallo- $\beta$ lactamase (Christopeit et al., 2016). Interestingly, 6,8-dichloroand 6,8-dibromo-3-formylchromones possess potent urease inhibitory activity, whereas 6-fluoro-, 6-chloro- and 6-bromo3-formylchromones exhibit no ability to inhibit urease (Kawase et al., 2007). Thus, the position of halogen atoms on the chromone ring should be associated with the urease inhibitory activity.<smiles>O=Cc1coc2cc(I)ccc2c1=O</smiles>

We have previously reported the crystal structures of 6,8-dichloro-4-oxochromene-3-carbaldehyde (6,8-dichloro-3formylchromone; Ishikawa \& Motohashi, 2013) and 6,8-dibromo-4-oxo-4H-chromene-3-carbaldehyde (6,8-dibromo-3formylchromone; Ishikawa, 2014a). In these crystals, halogen bonds are observed between the formyl oxygen atoms and the halogen atoms at the 8-position. Halogen bonding is defined as a net attractive interaction between an electrophilic region of a halogen atom in a molecule and a nucleophilic region of an atom in a molecule, and is characterized by a shorter contact between the two atoms. Halogen bonding has attracted much 


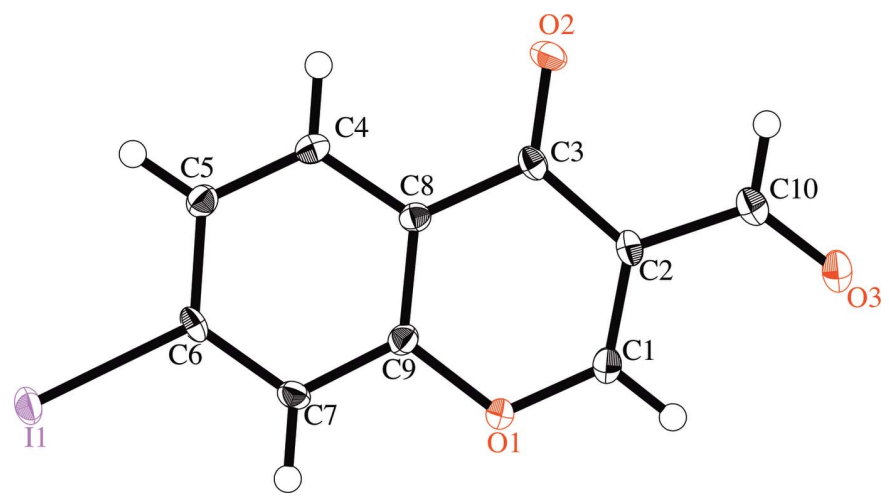

Figure 1

The molecular structure of the title compound, with displacement ellipsoids drawn at the $50 \%$ probability level. $\mathrm{H}$ atoms are shown as small spheres of arbitrary radius.

attention in medicinal chemistry, chemical biology, supramolecular chemistry and crystal engineering (Scholfield et al., 2013; Wilcken et al., 2013; Persch et al., 2015; Cavallo et al., 2016).

As part of an investigation of halogenated 3-formylchromones relevant to urease inhibitory activity and halogen bonding, I herein report the crystal structure of 7-iodo-4-oxo4H-chromene-3-carbaldehyde (7-iodo-3-formylchromone). The main objective of this study is to reveal the interaction mode of the iodine substituent at the 7-position of the chromone ring in the solid state.

\section{Structure commentary}

The mean deviation of the least-square planes for the nonhydrogen atoms is $0.0344 \AA$, and the largest deviation is 0.101 (3) $\AA$ for O3, indicating that these atoms are essentially coplanar (Fig. 1). All bond distances and angles are within their expected ranges.

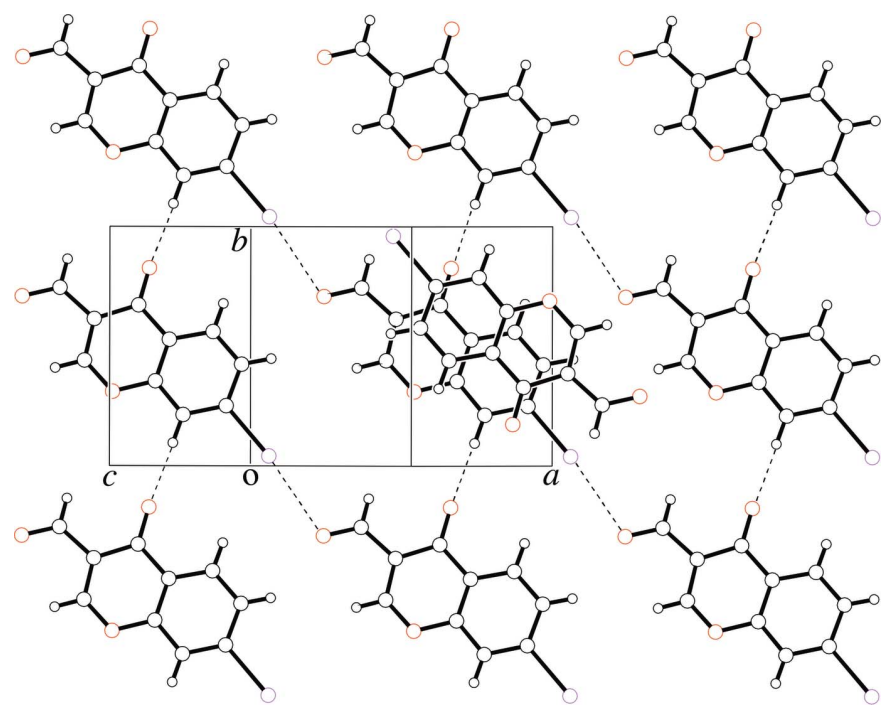

Figure 2

A packing view of the title compound. $\mathrm{C}-\mathrm{H} \cdots \mathrm{O}$ hydrogen bonds and $\mathrm{I}$... O halogen bonds are represented as dashed lines.
Table 1

Hydrogen-bond geometry $\left(\AA{ }^{\circ}{ }^{\circ}\right)$.

\begin{tabular}{lllll}
\hline$D-\mathrm{H} \cdots A$ & $D-\mathrm{H}$ & $\mathrm{H} \cdots A$ & $D \cdots A$ & $D-\mathrm{H} \cdots A$ \\
\hline $\mathrm{C} 7-\mathrm{H} 4 \cdots \mathrm{O} 2^{\mathrm{i}}$ & 0.95 & 2.35 & $3.208(4)$ & $150(1)$ \\
\hline
\end{tabular}

Symmetry code: (i) $x, y-1, z$.

\section{Supramolecular features}

In the crystal, the molecules are linked through $\pi-\pi$ stacking interactions between inversion-symmetry-equivalent ${ }^{\mathrm{i}}$ molecules [centroid-centroid distance between the benzene rings of the $4 H$-chromene units $=3.700$ (3) $\AA$; symmetry code: (i) $-x,-y,-z]$, and through $\mathrm{C}-\mathrm{H} \cdots \mathrm{O}$ hydrogen bonds (Table 1 ) that involve the $\mathrm{C} 7 / \mathrm{O} 2$ atoms. In particular, significant shorter contacts are observed between the iodine atoms and the formyl oxygen atoms of translation-symmetry equivalent ${ }^{\mathrm{ii}}$ molecules $\left[\mathrm{I} 1 \cdots \mathrm{O} 3=3.056\right.$ (2) $\AA$, C6-I1 $\cdots \mathrm{O} 3=173.18(8)^{\circ}$, $\mathrm{I} 1 \cdots \mathrm{O} 3-\mathrm{C} 10=111.12(18)^{\circ}$; symmetry code: (ii) $x+1, y-1$, $z$ ] along [ $\left.\begin{array}{lll}1 & 1 & 0\end{array}\right]$, resulting in sheets perpendicular to the $c$-axis, constructed by $\mathrm{C}-\mathrm{H} \cdots \mathrm{O}$ hydrogen bonds and $\mathrm{I} \cdots \mathrm{O}$ halogen bonds (Fig. 2).

\section{Database survey}

A search of WebCSD (Version 1.1.2, last update Oct 2016; Groom et al., 2014) for 7-halogeno-3-formylchromones gave the following three hits: 7-fluoro- (Asad et al., 2011), 7-chloro(Ishikawa, 2014b), and 7-bromo-3-formylchromone (Ishikawa, 2014c). In 7-fluoro-3-formylchromone, no contact around the fluorine atom is seen (Fig. $3 a$ ). In the crystals of 7-chloro- and 7-bromo-3-formylchromones, type I and type II halogen... halogen contacts are found, respectively (Fig. $3 b$ and $3 c$ ), and these halogen $\cdots$ halogen contacts are commonly found for $\mathrm{Cl}$ and $\mathrm{Br}$ atoms (Mukherjee et al., 2014). It should be noted that shorter contacts between oxygen atoms and halogen atoms are observed in 7-iodo-3-formylchromone (this work, Fig. 3d), but not in 7-fluoro-, 7-chloro-, and 7-bromo-3-formylchromones. This is in agreement with an assumption that the iodine atom should have the largest $\sigma$-hole (Clark et al., 2007) among the halogen atoms in 7-halogeno-3-formylchromones. These findings should be helpful in understanding the interaction of halogenated 3-formylchromones with urease, and is thus valuable for rational drug design.

\section{Synthesis and crystallization}

2'-Hydroxy-4'-iodoacetophenone was prepared from 3 -acetoxyiodobenzene by a Fries rearrangement reaction. To a solution of $2^{\prime}$-hydroxy-4'-iodoacetophenone $(4.4 \mathrm{mmol})$ in $N, N$-dimethylformamide $(10 \mathrm{ml})$ was added dropwise $\mathrm{POCl}_{3}$ $(13.2 \mathrm{mmol})$ at $273 \mathrm{~K}$. After the mixture was stirred for $14 \mathrm{~h}$ at room temperature, water $(100 \mathrm{ml})$ was added. The precipitates were collected, washed with water, and dried in vacuo at $333 \mathrm{~K}$ (yield 86\%). ${ }^{1} \mathrm{H}$ NMR (400 MHz, $\mathrm{CDCl}_{3}$ ): $\delta 7.84$ (dd, $1 \mathrm{H}, J=$ 8.8 and $1.5 \mathrm{~Hz}), 7.96(\mathrm{~d}, 1 \mathrm{H}, J=1.5 \mathrm{~Hz}), 7.99(\mathrm{~d}, 1 \mathrm{H}, J=$ 
(a)

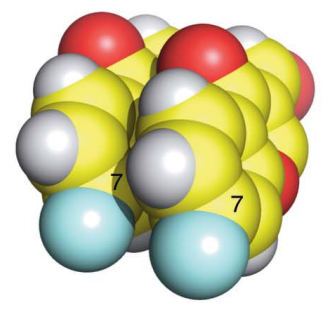

(b)

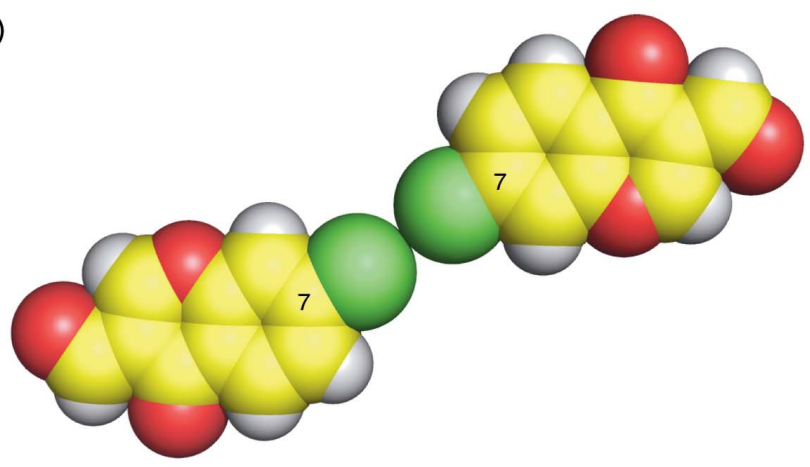

(c)
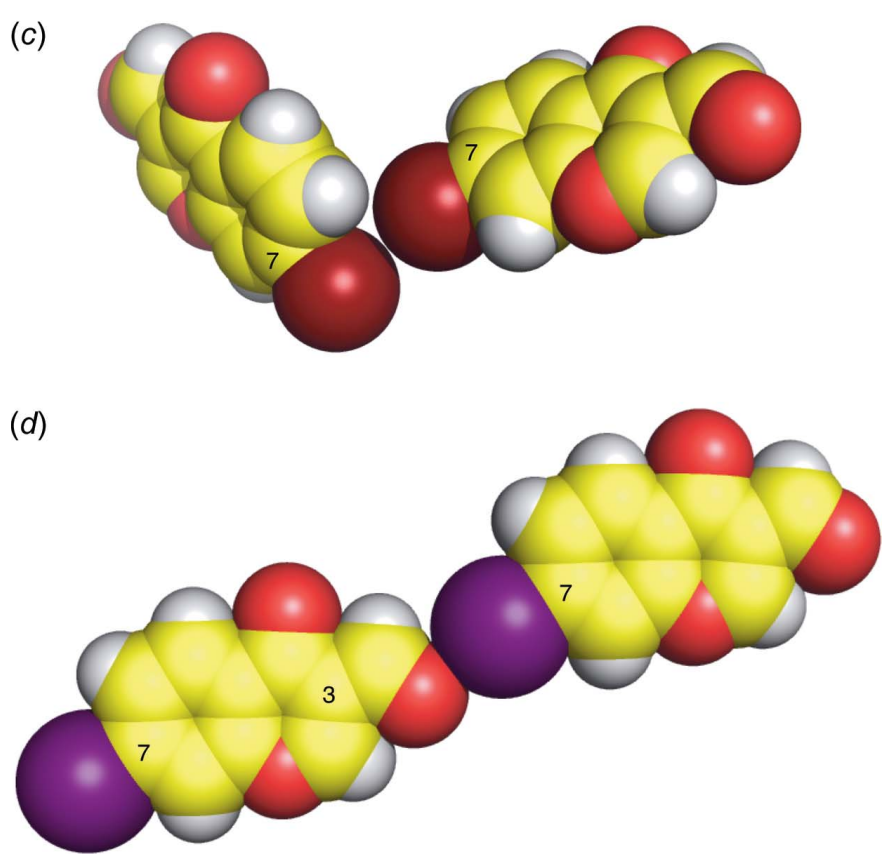

Figure 3

Sphere models of the crystal structures of (a) 7-fluoro-4-oxo-4Hchromene-3-carbaldehyde (Asad et al., 2011), (b) 7-chloro-4-oxo-4Hchromene-3-carbaldehyde (Ishikawa, 2014b), (c) 7-bromo-4-oxo-4Hchromene-3-carbaldehyde (Ishikawa, 2014c) and (d) the title compound (this work).

$8.3 \mathrm{~Hz}), 8.49$ (s, 1H), 10.36 (s, 1H). Single crystals suitable for $\mathrm{X}$-ray diffraction were obtained by slow evaporation of a 1,2dichloroethane solution of the title compound at room temperature.

\section{Refinement}

Crystal data, data collection and structure refinement details are summarized in Table 2. The C-bound hydrogen atoms
Table 2

Experimental details.

\begin{tabular}{|c|c|}
\hline \multicolumn{2}{|l|}{ Crystal data } \\
\hline Chemical formula & $\mathrm{C}_{10} \mathrm{H}_{5} \mathrm{IO}_{3}$ \\
\hline$M_{\mathrm{r}}$ & 300.05 \\
\hline Crystal system, space group & Monoclinic, $P 2_{1} / n$ \\
\hline Temperature $(\mathrm{K})$ & 100 \\
\hline$a, b, c(\AA)$ & $9.572(4), 7.533(4), 13.095(9)$ \\
\hline$\beta\left({ }^{\circ}\right)$ & $103.06(4)$ \\
\hline$V\left(\AA^{3}\right)$ & $919.8(9)$ \\
\hline$Z$ & 4 \\
\hline Radiation type & Мo $K \alpha$ \\
\hline$\mu\left(\mathrm{mm}^{-1}\right)$ & 3.46 \\
\hline Crystal size $(\mathrm{mm})$ & $0.25 \times 0.18 \times 0.15$ \\
\hline \multicolumn{2}{|l|}{ Data collection } \\
\hline Diffractometer & Rigaku AFC7R \\
\hline Absorption correction & $\psi$ scan (North et al., 1968) \\
\hline$T_{\min }, T_{\max }$ & $0.528,0.595$ \\
\hline $\begin{array}{l}\text { No. of measured, independent and } \\
\text { observed }\left[F^{2}>2.0 \sigma\left(F^{2}\right)\right] \text { reflec- } \\
\text { tions }\end{array}$ & $2538,2119,1916$ \\
\hline$R_{\text {int }}$ & 0.017 \\
\hline$(\sin \theta / \lambda)_{\max }\left(\AA^{-1}\right)$ & 0.650 \\
\hline \multicolumn{2}{|l|}{ Refinement } \\
\hline$R\left[F^{2}>2 \sigma\left(F^{2}\right)\right], w R\left(F^{2}\right), S$ & $0.021,0.053,1.06$ \\
\hline No. of reflections & 2119 \\
\hline No. of parameters & 128 \\
\hline H-atom treatment & $\mathrm{H}$-atom parameters constrained \\
\hline$\Delta \rho_{\max }, \Delta \rho_{\min }\left(\mathrm{e} \AA^{-3}\right)$ & $0.56,-0.60$ \\
\hline
\end{tabular}

Computer programs: WinAFC (Rigaku, 1999), SIR2011 (Burla et al., 2012), SHELXL2014 (Sheldrick, 2015) and CrystalStructure (Rigaku, 2015).

were placed in geometrical positions and refined using a riding model $\left[\mathrm{C}-\mathrm{H}=0.95 \AA\right.$ and $\left.U_{\text {iso }}(\mathrm{H})=1.2 U_{\text {eq }}(\mathrm{C})\right]$.

\section{Acknowledgements}

This work was supported by JSPS KAKENHI (Grant No. JP16K08199). I acknowledge University of Shizuoka for instrumental support.

\section{References}

Asad, M., Oo, C.-W., Osman, H., Hemamalini, M. \& Fun, H.-K. (2011). Acta Cryst. E67, o766.

Burla, M. C., Caliandro, R., Camalli, M., Carrozzini, B., Cascarano, G. L., Giacovazzo, C., Mallamo, M., Mazzone, A., Polidori, G. \& Spagna, R. (2012). J. Appl. Cryst. 45, 357-361.

Cavallo, G., Metrangolo, P., Milani, R., Pilati, T., Priimagi, A., Resnati, G. \& Terraneo, G. (2016). Chem. Rev. 116, 2478-2601.

Christopeit, T., Albert, A. \& Leiros, H. K. (2016). Bioorg. Med. Chem. 24, 2947-2953.

Clark, T., Hennemann, M., Murray, J. S. \& Politzer, P. (2007). J. Mol. Model. 13, 291-296.

Ekinci, D., Al-Rashida, M., Abbas, G., Şentürk, M. \& Supuran, C. T. (2012). J. Enzyme Inhib. Med. Chem. 27, 744-747.

Groom, C. R. \& Allen, F. H. (2014). Angew. Chem. Int. Ed. 53, 662671.

Ishikawa, Y. (2014a). Acta Cryst. E70, o439.

Ishikawa, Y. (2014b). Acta Cryst. E70, o831.

Ishikawa, Y. (2014c). Acta Cryst. E70, o996.

Ishikawa, Y. \& Motohashi, Y. (2013). Acta Cryst. E69, o1416.

Kawase, M., Tanaka, T., Kan, H., Tani, S., Nakashima, H. \& Sakagami, H. (2007). In Vivo, 21, 829-834.

Khan, K. M., Ambreen, N., Hussain, S., Perveen, S. \& Choudhary, M. I. (2009). Bioorg. Med. Chem. 17, 2983-2988. 
Khan, K. M., Ambreen, N., Mughal, U. R., Jalil, S., Perveen, S. \& Choudhary, M. I. (2010). Eur. J. Med. Chem. 45, 4058-4064.

Mukherjee, A. \& Desiraju, G. R. (2014). IUCrJ, 1, 49-60.

North, A. C. T., Phillips, D. C. \& Mathews, F. S. (1968). Acta Cryst. A24, 351-359.

Persch, E., Dumele, O. \& Diederich, F. (2015). Angew. Chem. Int. Ed. 54, 3290-3327.

Rigaku (1999). WinAFC Diffractometer Control Software. Rigaku Corporation, Tokyo, Japan.
Rigaku (2015). CrystalStructure. Rigaku Corporation, Tokyo, Japan. Scholfield, M. R., Zanden, C. M., Carter, M. \& Ho, P. S. (2013). Protein Sci. 22, 139-152.

Sheldrick, G. M. (2015). Acta Cryst. C71, 3-8.

Shim, Y. S., Kim, K. C., Lee, K. A., Shrestha, S., Lee, K. H., Kim, C. K. \& Cho, H. (2005). Bioorg. Med. Chem. 13, 1325-1332.

Wilcken, R., Zimmermann, M. O., Lange, A., Joerger, A. C. \& Boeckler, F. M. (2013). J. Med. Chem. 56, 1363-1388. 


\section{supporting information}

Acta Cryst. (2016). E72, 1724-1727 [https://doi.org/10.1107/S2056989016016972]

\section{Crystal structure of 7-iodo-4-oxo-4H-chromene-3-carbaldehyde}

\section{Yoshinobu Ishikawa}

\section{Computing details}

Data collection: WinAFC (Rigaku, 1999); cell refinement: WinAFC (Rigaku, 1999); data reduction: WinAFC (Rigaku, 1999); program(s) used to solve structure: SIR2011 (Burla et al., 2012); program(s) used to refine structure: SHELXL2014 (Sheldrick, 2015); molecular graphics: CrystalStructure (Rigaku, 2015); software used to prepare material for publication: CrystalStructure (Rigaku, 2015).

7-Iodo-4-oxo-4H-chromene-3-carbaldehyde

Crystal data

$\mathrm{C}_{10} \mathrm{H}_{5} \mathrm{IO}_{3}$

$M_{r}=300.05$

Monoclinic, $P 2_{1} / n$

$a=9.572(4) \AA$

$b=7.533$ (4) $\AA$

$c=13.095(9) \AA$

$\beta=103.06(4)^{\circ}$

$V=919.8(9) \AA^{3}$

$Z=4$

Data collection

Rigaku AFC7R diffractometer

$\omega-2 \theta$ scans

Absorption correction: $\psi$ scan

(North et al., 1968)

$T_{\min }=0.528, T_{\max }=0.595$

2538 measured reflections

2119 independent reflections

\section{Refinement}

Refinement on $F^{2}$

$R\left[F^{2}>2 \sigma\left(F^{2}\right)\right]=0.021$

$w R\left(F^{2}\right)=0.053$

$S=1.06$

2119 reflections

128 parameters

0 restraints

Primary atom site location: structure-invariant direct methods

Secondary atom site location: difference Fourier map
$F(000)=568.00$

$D_{\mathrm{x}}=2.167 \mathrm{Mg} \mathrm{m}^{-3}$

Mo $K \alpha$ radiation, $\lambda=0.71069 \AA$

Cell parameters from 25 reflections

$\theta=15.1-17.1^{\circ}$

$\mu=3.46 \mathrm{~mm}^{-1}$

$T=100 \mathrm{~K}$

Prismatic, yellow

$0.25 \times 0.18 \times 0.15 \mathrm{~mm}$

1916 reflections with $F^{2}>2.0 \sigma\left(F^{2}\right)$

$R_{\text {int }}=0.017$

$\theta_{\max }=27.5^{\circ}, \theta_{\min }=3.0^{\circ}$

$h=-12 \rightarrow 12$

$k=-9 \rightarrow 0$

$l=-9 \rightarrow 17$

3 standard reflections every 150 reflections intensity decay: $-0.3 \%$

Hydrogen site location: inferred from neighbouring sites

$\mathrm{H}$-atom parameters constrained

$w=1 /\left[\sigma^{2}\left(F_{\mathrm{o}}{ }^{2}\right)+(0.0244 P)^{2}+1.8229 P\right]$

where $P=\left(F_{\mathrm{o}}^{2}+2 F_{\mathrm{c}}^{2}\right) / 3$

$(\Delta / \sigma)_{\max }=0.001$

$\Delta \rho_{\max }=0.56{\mathrm{e} \AA^{-3}}^{-3}$

$\Delta \rho_{\text {min }}=-0.60$ e $\AA^{-3}$

Extinction correction: SHELXL2014

(Sheldrick, 2015)

Extinction coefficient: 0.0024 (4) 


\section{Special details}

Geometry. All esds (except the esd in the dihedral angle between two 1.s. planes) are estimated using the full covariance matrix. The cell esds are taken into account individually in the estimation of esds in distances, angles and torsion angles; correlations between esds in cell parameters are only used when they are defined by crystal symmetry. An approximate (isotropic) treatment of cell esds is used for estimating esds involving l.s. planes.

Refinement. Refinement was performed using all reflections. The weighted $R$-factor $(w R)$ and goodness of fit $(S)$ are based on $F^{2}$. R-factor (gt) are based on $F$. The threshold expression of $F^{2}>2.0 \sigma\left(F^{2}\right)$ is used only for calculating $R$-factor (gt).

Fractional atomic coordinates and isotropic or equivalent isotropic displacement parameters $\left(\AA^{2}\right)$

\begin{tabular}{lllll}
\hline & $x$ & $y$ & $z$ & $U_{\text {iso }} * / U_{\text {eq }}$ \\
\hline I1 & $0.24361(2)$ & $0.04012(2)$ & $0.39087(2)$ & $0.01629(8)$ \\
O1 & $-0.2715(2)$ & $0.3092(3)$ & $0.39728(15)$ & $0.0152(4)$ \\
O2 & $-0.1775(2)$ & $0.8253(3)$ & $0.33965(16)$ & $0.0172(4)$ \\
O3 & $-0.5743(2)$ & $0.7067(3)$ & $0.39144(17)$ & $0.0209(4)$ \\
C1 & $-0.3676(3)$ & $0.4391(4)$ & $0.3915(2)$ & $0.0160(5)$ \\
H1 & -0.4603 & 0.4076 & 0.4004 & $0.019^{*}$ \\
C2 & $-0.3435(3)$ & $0.6125(4)$ & $0.3740(2)$ & $0.0135(5)$ \\
C3 & $-0.2044(3)$ & $0.6703(4)$ & $0.3577(2)$ & $0.0128(5)$ \\
C4 & $0.0421(3)$ & $0.5595(4)$ & $0.3565(2)$ & $0.0143(5)$ \\
H2 & 0.0697 & 0.6769 & 0.3434 & $0.017^{*}$ \\
C5 & $0.1408(3)$ & $0.4232(4)$ & $0.3655(2)$ & $0.0144(5)$ \\
H3 & 0.2365 & 0.4468 & 0.3604 & $0.017^{*}$ \\
C6 & $0.0979(3)$ & $0.2499(4)$ & $0.3822(2)$ & $0.0133(5)$ \\
C7 & $-0.0402(3)$ & $0.2124(4)$ & $0.3922(2)$ & $0.0135(5)$ \\
H4 & -0.0685 & 0.0945 & 0.4039 & $0.016^{*}$ \\
C8 & $-0.0982(3)$ & $0.5267(4)$ & $0.3665(2)$ & $0.0123(5)$ \\
C9 & $-0.1358(3)$ & $0.3530(4)$ & $0.3847(2)$ & $0.0132(5)$ \\
C10 & $-0.4587(3)$ & $0.7427(4)$ & $0.3717(2)$ & $0.0162(5)$ \\
H5 & -0.4422 & 0.8619 & 0.3538 & $0.019^{*}$ \\
& & & &
\end{tabular}

Atomic displacement parameters $\left(\AA^{2}\right)$

\begin{tabular}{lllllll}
\hline & $U^{11}$ & $U^{22}$ & $U^{33}$ & $U^{12}$ & $U^{13}$ & $U^{23}$ \\
\hline I1 & $0.01410(10)$ & $0.01533(11)$ & $0.01997(11)$ & $0.00472(7)$ & $0.00501(7)$ & $-0.00105(7)$ \\
O1 & $0.0119(9)$ & $0.0131(9)$ & $0.0217(10)$ & $0.0010(7)$ & $0.0064(8)$ & $0.0019(8)$ \\
O2 & $0.0186(10)$ & $0.0101(9)$ & $0.0221(10)$ & $-0.0001(8)$ & $0.0029(8)$ & $0.0011(8)$ \\
O3 & $0.0155(10)$ & $0.0215(11)$ & $0.0263(11)$ & $0.0054(8)$ & $0.0064(8)$ & $0.0012(9)$ \\
C1 & $0.0120(12)$ & $0.0173(14)$ & $0.0190(13)$ & $0.0022(10)$ & $0.0042(10)$ & $0.0010(11)$ \\
C2 & $0.0119(12)$ & $0.0149(13)$ & $0.0135(12)$ & $0.0041(10)$ & $0.0020(10)$ & $0.0009(11)$ \\
C3 & $0.0132(12)$ & $0.0126(12)$ & $0.0115(12)$ & $0.0031(10)$ & $0.0001(9)$ & $-0.0006(10)$ \\
C4 & $0.0139(12)$ & $0.0140(13)$ & $0.0149(12)$ & $-0.0029(10)$ & $0.0029(10)$ & $-0.0004(10)$ \\
C5 & $0.0125(12)$ & $0.0151(14)$ & $0.0156(13)$ & $-0.0014(10)$ & $0.0033(10)$ & $-0.0017(10)$ \\
C6 & $0.0154(12)$ & $0.0111(12)$ & $0.0137(12)$ & $0.0051(10)$ & $0.0035(10)$ & $-0.0025(10)$ \\
C7 & $0.0154(12)$ & $0.0093(12)$ & $0.0154(12)$ & $-0.0005(10)$ & $0.0027(10)$ & $-0.0014(10)$ \\
C8 & $0.0129(12)$ & $0.0119(12)$ & $0.0121(12)$ & $-0.0013(10)$ & $0.0026(9)$ & $-0.0010(10)$ \\
C9 & $0.0121(12)$ & $0.0142(13)$ & $0.0140(12)$ & $-0.0008(10)$ & $0.0044(10)$ & $0.0001(10)$
\end{tabular}




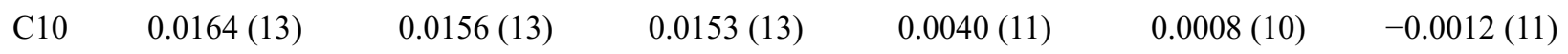

Geometric parameters $(\AA, \stackrel{o}{)}$

\begin{tabular}{|c|c|c|c|}
\hline $\mathrm{I} 1-\mathrm{C} 6$ & $2.094(3)$ & $\mathrm{C} 4-\mathrm{C} 5$ & $1.383(4)$ \\
\hline $\mathrm{O} 1-\mathrm{C} 1$ & $1.334(3)$ & $\mathrm{C} 4-\mathrm{C} 8$ & $1.400(4)$ \\
\hline $\mathrm{O} 1-\mathrm{C} 9$ & $1.386(3)$ & $\mathrm{C} 4-\mathrm{H} 2$ & 0.9500 \\
\hline $\mathrm{O} 2-\mathrm{C} 3$ & $1.230(3)$ & $\mathrm{C} 5-\mathrm{C} 6$ & $1.401(4)$ \\
\hline $\mathrm{O} 3-\mathrm{C} 10$ & $1.222(4)$ & $\mathrm{C} 5-\mathrm{H} 3$ & 0.9500 \\
\hline $\mathrm{C} 1-\mathrm{C} 2$ & $1.355(4)$ & $\mathrm{C} 6-\mathrm{C} 7$ & $1.387(4)$ \\
\hline $\mathrm{C} 1-\mathrm{H} 1$ & 0.9500 & $\mathrm{C} 7-\mathrm{C} 9$ & $1.389(4)$ \\
\hline $\mathrm{C} 2-\mathrm{C} 3$ & $1.462(4)$ & $\mathrm{C} 7-\mathrm{H} 4$ & 0.9500 \\
\hline $\mathrm{C} 2-\mathrm{C} 10$ & $1.471(4)$ & $\mathrm{C} 8-\mathrm{C} 9$ & $1.392(4)$ \\
\hline $\mathrm{C} 3-\mathrm{C} 8$ & $1.471(4)$ & $\mathrm{C} 10-\mathrm{H} 5$ & 0.9500 \\
\hline $\mathrm{C} 1-\mathrm{O} 1-\mathrm{C} 9$ & $118.2(2)$ & $\mathrm{C} 7-\mathrm{C} 6-\mathrm{C} 5$ & $121.6(2)$ \\
\hline $\mathrm{O} 1-\mathrm{C} 1-\mathrm{C} 2$ & $125.1(3)$ & $\mathrm{C} 7-\mathrm{C} 6-\mathrm{I} 1$ & $118.6(2)$ \\
\hline $\mathrm{O} 1-\mathrm{C} 1-\mathrm{H} 1$ & 117.4 & $\mathrm{C} 5-\mathrm{C} 6-\mathrm{I} 1$ & $119.8(2)$ \\
\hline $\mathrm{C} 2-\mathrm{C} 1-\mathrm{H} 1$ & 117.4 & $\mathrm{C} 6-\mathrm{C} 7-\mathrm{C} 9$ & $117.7(3)$ \\
\hline $\mathrm{C} 1-\mathrm{C} 2-\mathrm{C} 3$ & $120.5(2)$ & $\mathrm{C} 6-\mathrm{C} 7-\mathrm{H} 4$ & 121.2 \\
\hline $\mathrm{C} 1-\mathrm{C} 2-\mathrm{C} 10$ & $119.4(3)$ & $\mathrm{C} 9-\mathrm{C} 7-\mathrm{H} 4$ & 121.2 \\
\hline $\mathrm{C} 3-\mathrm{C} 2-\mathrm{C} 10$ & $120.1(3)$ & $\mathrm{C} 9-\mathrm{C} 8-\mathrm{C} 4$ & $118.2(2)$ \\
\hline $\mathrm{O} 2-\mathrm{C} 3-\mathrm{C} 2$ & $123.2(3)$ & $\mathrm{C} 9-\mathrm{C} 8-\mathrm{C} 3$ & $120.2(2)$ \\
\hline $\mathrm{O} 2-\mathrm{C} 3-\mathrm{C} 8$ & $122.8(2)$ & $\mathrm{C} 4-\mathrm{C} 8-\mathrm{C} 3$ & $121.5(3)$ \\
\hline $\mathrm{C} 2-\mathrm{C} 3-\mathrm{C} 8$ & $113.9(2)$ & $\mathrm{O} 1-\mathrm{C} 9-\mathrm{C} 7$ & $115.5(2)$ \\
\hline $\mathrm{C} 5-\mathrm{C} 4-\mathrm{C} 8$ & $120.8(3)$ & $\mathrm{O} 1-\mathrm{C} 9-\mathrm{C} 8$ & $122.0(2)$ \\
\hline $\mathrm{C} 5-\mathrm{C} 4-\mathrm{H} 2$ & 119.6 & $\mathrm{C} 7-\mathrm{C} 9-\mathrm{C} 8$ & $122.5(2)$ \\
\hline $\mathrm{C} 8-\mathrm{C} 4-\mathrm{H} 2$ & 119.6 & $\mathrm{O} 3-\mathrm{C} 10-\mathrm{C} 2$ & $123.9(3)$ \\
\hline $\mathrm{C} 4-\mathrm{C} 5-\mathrm{C} 6$ & $119.1(3)$ & $\mathrm{O} 3-\mathrm{C} 10-\mathrm{H} 5$ & 118.0 \\
\hline $\mathrm{C} 4-\mathrm{C} 5-\mathrm{H} 3$ & 120.5 & $\mathrm{C} 2-\mathrm{C} 10-\mathrm{H} 5$ & 118.0 \\
\hline $\mathrm{C} 6-\mathrm{C} 5-\mathrm{H} 3$ & 120.5 & & \\
\hline $\mathrm{C} 9-\mathrm{O} 1-\mathrm{C} 1-\mathrm{C} 2$ & $0.1(4)$ & $\mathrm{O} 2-\mathrm{C} 3-\mathrm{C} 8-\mathrm{C} 9$ & $-178.5(3)$ \\
\hline $\mathrm{O} 1-\mathrm{C} 1-\mathrm{C} 2-\mathrm{C} 3$ & $1.1(4)$ & $\mathrm{C} 2-\mathrm{C} 3-\mathrm{C} 8-\mathrm{C} 9$ & $2.4(4)$ \\
\hline $\mathrm{O} 1-\mathrm{C} 1-\mathrm{C} 2-\mathrm{C} 10$ & $-178.8(2)$ & $\mathrm{O} 2-\mathrm{C} 3-\mathrm{C} 8-\mathrm{C} 4$ & $1.2(4)$ \\
\hline $\mathrm{C} 1-\mathrm{C} 2-\mathrm{C} 3-\mathrm{O} 2$ & $178.6(3)$ & $\mathrm{C} 2-\mathrm{C} 3-\mathrm{C} 8-\mathrm{C} 4$ & $-177.9(2)$ \\
\hline $\mathrm{C} 10-\mathrm{C} 2-\mathrm{C} 3-\mathrm{O} 2$ & $-1.5(4)$ & $\mathrm{C} 1-\mathrm{O} 1-\mathrm{C} 9-\mathrm{C} 7$ & $179.6(2)$ \\
\hline $\mathrm{C} 1-\mathrm{C} 2-\mathrm{C} 3-\mathrm{C} 8$ & $-2.3(4)$ & $\mathrm{C} 1-\mathrm{O} 1-\mathrm{C} 9-\mathrm{C} 8$ & $0.0(4)$ \\
\hline $\mathrm{C} 10-\mathrm{C} 2-\mathrm{C} 3-\mathrm{C} 8$ & $177.6(2)$ & $\mathrm{C} 6-\mathrm{C} 7-\mathrm{C} 9-\mathrm{O} 1$ & $-178.8(2)$ \\
\hline $\mathrm{C} 8-\mathrm{C} 4-\mathrm{C} 5-\mathrm{C} 6$ & $1.6(4)$ & $\mathrm{C} 6-\mathrm{C} 7-\mathrm{C} 9-\mathrm{C} 8$ & $0.7(4)$ \\
\hline $\mathrm{C} 4-\mathrm{C} 5-\mathrm{C} 6-\mathrm{C} 7$ & $-1.5(4)$ & $\mathrm{C} 4-\mathrm{C} 8-\mathrm{C} 9-\mathrm{O} 1$ & $179.0(2)$ \\
\hline $\mathrm{C} 4-\mathrm{C} 5-\mathrm{C} 6-\mathrm{I} 1$ & $177.9(2)$ & $\mathrm{C} 3-\mathrm{C} 8-\mathrm{C} 9-\mathrm{O} 1$ & $-1.4(4)$ \\
\hline $\mathrm{C} 5-\mathrm{C} 6-\mathrm{C} 7-\mathrm{C} 9$ & $0.3(4)$ & $\mathrm{C} 4-\mathrm{C} 8-\mathrm{C} 9-\mathrm{C} 7$ & $-0.6(4)$ \\
\hline $\mathrm{I} 1-\mathrm{C} 6-\mathrm{C} 7-\mathrm{C} 9$ & $-179.1(2)$ & $\mathrm{C} 3-\mathrm{C} 8-\mathrm{C} 9-\mathrm{C} 7$ & $179.1(2)$ \\
\hline $\mathrm{C} 5-\mathrm{C} 4-\mathrm{C} 8-\mathrm{C} 9$ & $-0.6(4)$ & $\mathrm{C} 1-\mathrm{C} 2-\mathrm{C} 10-\mathrm{O} 3$ & $4.9(4)$ \\
\hline $\mathrm{C} 5-\mathrm{C} 4-\mathrm{C} 8-\mathrm{C} 3$ & $179.7(2)$ & $\mathrm{C} 3-\mathrm{C} 2-\mathrm{C} 10-\mathrm{O} 3$ & $-175.0(3)$ \\
\hline
\end{tabular}


Hydrogen-bond geometry $\left(\AA,{ }^{\circ}\right)$

\begin{tabular}{lllll}
\hline$D-\mathrm{H} \cdots A$ & $D-\mathrm{H}$ & $\mathrm{H} \cdots A$ & $D \cdots A$ & $D-\mathrm{H}^{\cdots} \cdot A$ \\
\hline $\mathrm{C} 7-\mathrm{H} 4 \cdots \mathrm{O}^{\mathrm{i}}$ & 0.95 & 2.35 & $3.208(4)$ & $150(1)$ \\
\hline
\end{tabular}

Symmetry code: (i) $x, y-1, z$. 